\title{
A Computational Geometry-based Local Search Algorithm for Planar Location Problems ${ }^{\star}$
}

\author{
Hadrien Cambazard, Deepak Mehta, Barry O'Sullivan, and Luis Quesada \\ CTVR, Cork Constraint Computation Centre, University College Cork, Ireland \\ $\{$ d.mehta|b.osullivan|l.quesada\}@4c.ucc.ie \\ Laboratoire G-SCOP, Grenoble INP-UJF-CNRS, 46 avenue Félix Viallet, 38031 \\ Grenoble, France \\ hadrien. cambazard@grenoble-inp.fr
}

\begin{abstract}
Constraint-based local search is an important paradigm in the field of constraint programming, particularly when considering very large optimisation problems. We are motivated by applications in areas such as telecommunications network design, warehouse location and other problems in which we wish to select an optimal set of locations from a two dimensional plane. The problems we are interested in are so large that they are ideal candidates for constraint-based local search methods. Maintaining the objective function incrementally is often a key element for efficient local search algorithms. In the case of two dimensional plane problems, we can often achieve incrementality by exploiting computational geometry. In this paper we present a novel approach to solving a class of placement problems for which Voronoi cell computation can provide an efficient form of incrementality. We present empirical results demonstrating the utility of our approach against the current state of the art.
\end{abstract}

\section{Introduction}

We are motivated by applications in areas such as telecommunications network design, warehouse location and other problems in which we wish to select an optimal set of locations from a two dimensional plane. Local search algorithms have proved very efficient in this area and we are interested in the design of efficient incremental mechanisms involving closest point problems. We investigate a restriction of this class of problems in which the cost of allocating a client to a facility is assumed to be proportional to Euclidean distance. This assumption initially comes from a practical problem encountered by the authors and focused on the design of resilient long-reach passive optical networks [10]. This restriction is however relevant in other application domains, in particular spatial clustering problems such as the one tackled in [12]. The core problem in this application ${ }^{1}$ is referred to as the Single Coverage Problem and defined as follows:

* This work is supported by Science Foundation Ireland Grant No. 10/CE/I1853.

${ }^{1}$ We simply present the core problem (which remains NP-complete even if the other constraints of the real application are not considered) to facilitate the process of establishing connections between our problem and problems studied in the literature. 
Definition 1 (The Single Coverage problem). Given a constant $\kappa$ and $a$ set $S=\left\{p_{i} \mid 1 \leq i \leq m\right\}$ of points in a two dimensional space where each point $p_{i}$ is associated with a weight $b_{i}$, the Single Coverage problem (SCP) is to decide whether there exists a set $W \subset S$ of cardinality $p$ such that the weighted sum of the distances from the points in $S-W$ to their closest points in $W$ is less than or equal to $\kappa$, i.e., $\sum_{p_{i} \in S-W} b_{i} \times \min _{p_{j} \in W} d_{i j} \leq \kappa$.

$S$ is referred to as the set of clients and $W$ as the set of facilities. Moreover, $d_{i j}$ denotes the Euclidean distance between the points $p_{i}$ and $p_{j}$. The single coverage problem is strongly related to location problems in a plane such as the uncapacitated warehouse location problem (UWLP) [5]. Typically, the SCP differs from the UWLP in three respects: the transportation cost is proportional to the Euclidean distance (although this is usually the case in practice, UWLP does not make any assumption about the transportation costs); there is no fixed cost of opening a warehouse; and the number of opened warehouses is bounded by $p$. Another, and even closer, problem is the p-median problem [8]. This latter problem only differs from SCP because the similarity or dissimilarity measure used by p-median problems is not restricted to the Euclidean distance. A vast literature deals with location problems in the plane, the survey [6] presents spatial clustering problems.

State of the art algorithms for solving large-scale p-median (k-medoid) or uncapacitated warehouse location problems rely on local search. Genetic algorithms [9], and tabu search in particular have been very successful [11]. We describe in Sections 2 and 3 a local search algorithm for the single coverage problem directly inspired by this previous work. In particular the incrementality of the neighborhood has been described in detail $[11,16,15,1]$. In Section 4 , we present a novel approach to improving the complexity of the incremental algorithms presented in the previous section when the cost is proportional to Euclidean distance. We present the empirical evaluation in Section 6. The approaches are evaluated using two sets of experiments. In the first set of experiments we investigated the behavior of the proposed algorithms on randomly generated data, particularly focusing on benchmarking the primitive operations. In the second set, we considered eight SCP cases where half of them corresponded to real cases coming from network operators in Ireland and UK and the other half corresponded to cases randomly generated. Our experimental evaluation confirmed the superiority observed in the complexity analysis.

\section{The Tabu Search}

We denote the set of current facilities by $W$ and the current set of clients/nodes by $C=S-W$. We use $i$ as an index for clients whereas $j$ refers to facilities.

Neighborhood. The neighborhood is defined by moving a facility from one location to another. This move is performed in two steps, a facility is closed first and another one is opened. Closing a facility involves removing a point $p_{j}$ from $W$. 
Opening a facility involves adding a point $p_{i}$ from $C$ to $W$. The objective function is evaluated by ensuring that clients are always connected to their closest facilities. This invariant is maintained throughout the search. This neighborhood is used by the main algorithm for $k$-medoid, namely the Partitioning Around Medoids (PAM) algorithm [8]. However, PAM does not include an incremental evaluation of the moves. Therefore we distinguish the two steps (opening and closing) to develop an incremental scheme using [11] for each of these steps separately. This incremental evaluation of the moves is achieved by maintaining $\Delta_{i}^{+}$ (the variation of cost due to adding $p_{i}$ in $W$ (opening a facility)) and $\Delta_{i}^{-}$(the variation of cost due to removing $p_{i}$ from $W$ (closing a facility)).

Search. The initial $p$ facilities are chosen randomly. The tabu mechanism is very simple. It prevents a point that was a facility in the last $t$ iterations, where $t$ is the length of the tabu-list, from becoming a facility again. The tabu-list is denoted $T$ in Algorithm 1. The first improving move found is performed. If no improving move exists, the facility to close is chosen randomly and the new best location for this facility is opened. After a number of non-improving iterations, the search is restarted from $p$ random facilities.

Algorithm 1 presents the general scheme of the tabu search. It assumes that two methods are available for opening and closing a facility (resp. OpenFacility and CloseFacility) while incrementally maintaining the value of the objective function (denoted $o b j$ ) and $\Delta^{+}$. It is not useful to maintain $\Delta^{-}$incrementally for this specific neighborhood, where all opened nodes are closed to evaluate each move (Line 8 is only using $\Delta^{+}$). $\Delta^{-}$would be useful in a more general context when the closing and opening operations can be done independently, e.g., warehouse location. This algorithm is very like the PAM algorithm; the only difference would be that PAM is selecting the best move rather than the first improving one. However our algorithm is enhanced with the incremental mechanisms, and the tabu metaheuristic, introduced in warehouse location for a similar neighborhood. We believe it is the best starting point for our application and purpose.

\section{Incremental Neighborhood}

Maintaining the objective function incrementally is a key element for efficient local search algorithms [11]. When moving a facility from one location to another, only a small subset of the clients are reallocated: clients that have lost their current closest facilities and clients that have gained a new closest facility. The cost is not affected by the other clients. Optimal incremental algorithms have been published for opening and closing operations in the context of warehouse location $[16,11,15]$. We present [11] in detail as we will build upon it. The data structures needed to develop the incremental approach and maintain $\Delta^{+}$and $\Delta^{-}$ are the following: $a_{i}^{1}$ is the closest facility to client $i$ so that $a_{i}^{1}=\arg \min _{p_{j} \in W} d_{i j}$; $a_{i}^{2}$ is the second closest facility to client $i$ so that $a_{i}^{2}=\arg \min _{p_{j} \in W \mid p_{j} \neq a_{i}^{1}} d_{i j}$; $Q_{i}$ is a priority queue storing all the current facilities $W$ ordered by increasing distance from $i$. Consequently $a_{i}^{1}$ and $a_{i}^{2}$ are the first two elements of this queue. 


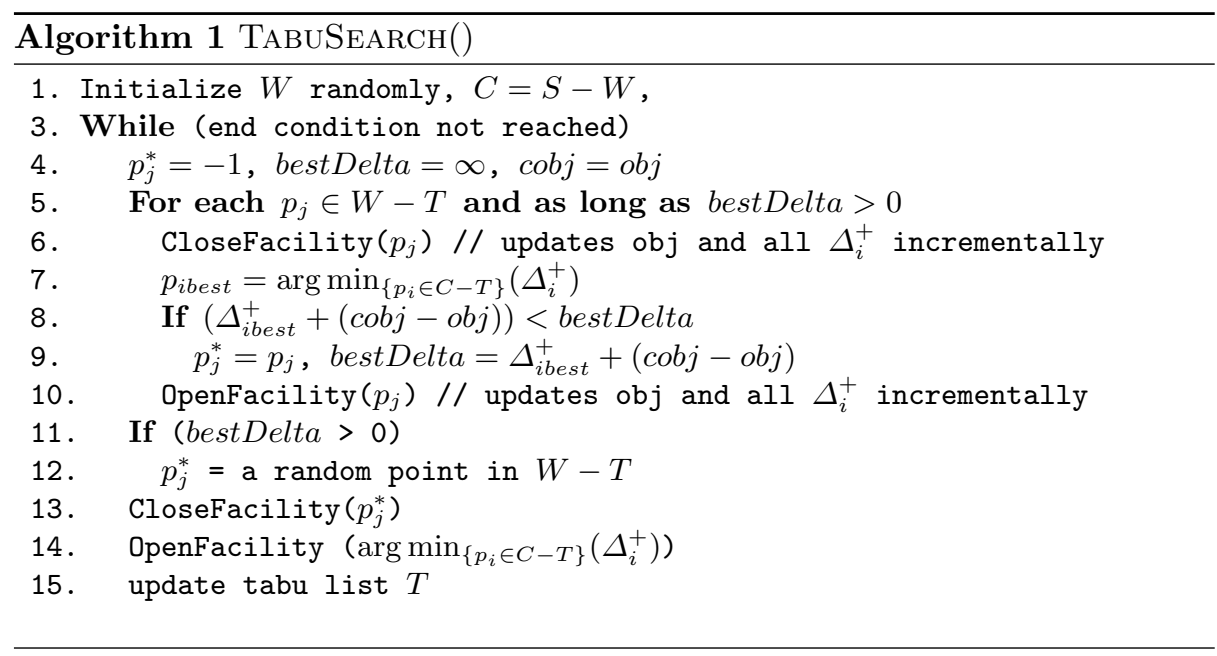

The variations of the objective function due to closing and opening a facility $i$ are initialized as follows:

$$
\begin{aligned}
\Delta_{i}^{-} & =\sum_{p_{j} \in S \mid a_{j}^{1}=p_{i}} b_{j} \times\left(d_{j, a_{j}^{2}}-d_{j i}\right) \\
\Delta_{i}^{+} & =-\sum_{p_{j} \in C} b_{j} \times \max \left(0, d_{j, a_{j}^{1}}-d_{j i}\right)
\end{aligned}
$$

When closing a facility $i$, we need to add to the objective function the cost of disconnecting each point connected to $p_{i}$ and re-connecting them to their second closest facility. Therefore, we add $d_{j, a_{j}^{2}}$ and remove $d_{j i}$. Similarly when opening a facility $i$, each point $p_{j}$ of $C$ that is closer to this new facility than to its current closest facility $\left(d_{j, a_{j}^{1}}>d_{j i}\right)$ needs to be disconnected and re-connected decreasing the objective function by the quantity $d_{j, a_{j}^{1}}-d_{j i}$. Notice that $\Delta_{i}^{+}$is at most zero (opening never degrades the overall objective function) and $\Delta_{i}^{-}$is at least zero (closing always degrades the overall objective function). In what follows we will refer to $d_{j, a_{j}^{2}}-d_{j i}$ as the contribution of $p_{j}$ by which $\Delta_{i}^{-}$increases. Similarly, we will say that $d_{j, a_{j}^{1}}-d_{j i}$ is the contribution of $p_{j}$ by which $\Delta_{i}^{+}$decreases. For the sake of clarity we will assume that all the $b_{i}$ are equal to 1 in the following; the algorithms presented remain identical with general weights. It is simply a matter of multiplying the distance by the weight.

\subsection{Closing a Facility}

Algorithm 2 presents the incremental maintenance of the data structures, in particular $\Delta^{+}$and $\Delta^{-}$, when closing a given facility $p_{j}$.

For each client $p_{i}$ of $C$, the priority queue, $Q_{i}$, is maintained (Line 2). The previous values of the closest and second closest facilities of $p_{i}$ are saved in $o_{i}^{1}$ 


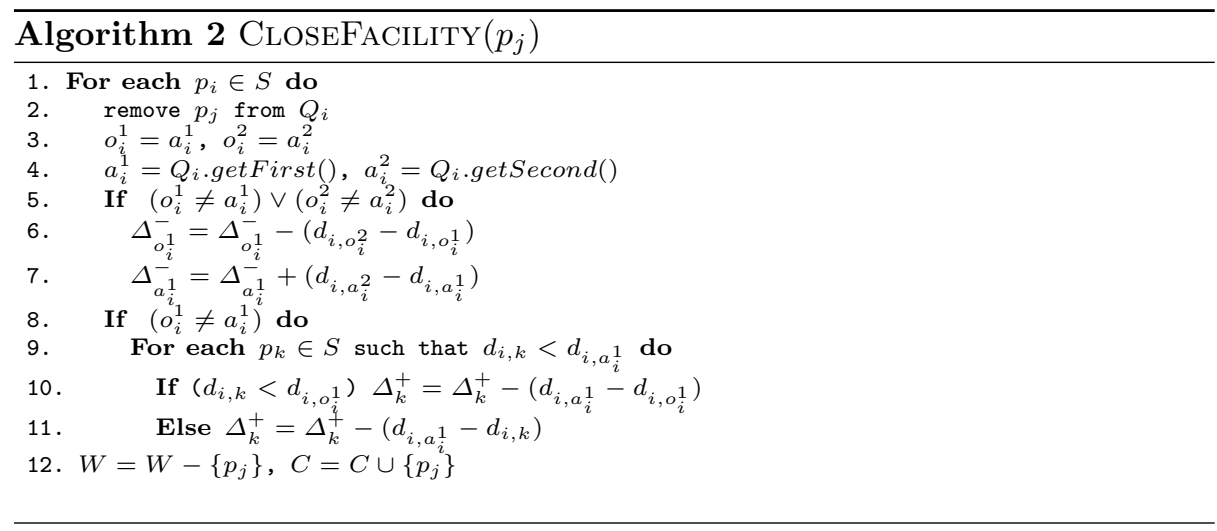

and $o_{i}^{2}$ respectively (Line 3 ). The closest and second closest facilities of $p_{i}$ are then updated in $a_{i}^{1}$ and $a_{i}^{2}$ using $Q_{i}$ respectively (Line 4). Lines 5 to 11 deal with the update of $\Delta^{+}$and $\Delta^{-}$. When a facility $p_{j}$ is closed either the closest facility of $p_{i}$ can change, or the second closest facility of $p_{i}$ can change, or none of them changes. Only the points $p_{i}$ which have a new closest or second closest facility can trigger the changes of the values of $\Delta^{-}$. Line 6 simply removes from $\Delta^{-}$the previous contribution of $p_{i}$ to its old closest facility and Line 7 adds the new contribution of $p_{i}$ to its new closest facility.

Lines $(8-11)$ update $\Delta_{k}^{+}$with respect to the contribution of $i$. From Equation (2) recall that the contribution of $i$ for $\Delta_{k}^{+}$can change only when the closest facility of $i$ changes, i.e., when $o_{i}^{1} \neq a_{i}^{1}$ (Line 8) and when $d_{i, k}<d_{i, a_{i}^{1}}$ (Line 9). Therefore, the iteration is performed on a pre-computed list of points $k$ sorted by distance from $i$ as long as the criteria $d_{i, k}<d_{i, a_{i}^{1}}$ holds. If $k$ is closer to $i$ than $o_{i}^{1}$ (i.e., $d_{i, k}<d_{i, o_{i}^{1}}$ ), as shown in Figure $1(\mathrm{a})$, then it follows that the contribution of $i$ to $\Delta_{k}^{+}$is non-zero. Therefore, the previous contribution, $d_{i, o_{i}^{1}}-d_{i, k}$, should be replaced by the new contribution $d_{i, a_{i}^{1}}-d_{i, k}$, which is effectively the difference between $d_{i, a_{i}^{1}}-d_{i, o_{i}^{1}}$ (Line 10). If $k$ is not closer to $i$ than $o_{i}^{1}$ as shown in Figure 1(b) then the contribution of $i$ to $\Delta_{k}^{+}$is 0 . Therefore, $\Delta_{k}^{+}$is updated with the new contribution of $i$ (Line 11).

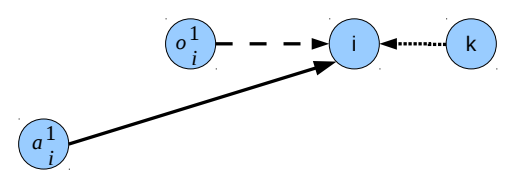

(a) $i$ was already contributing to $\Delta_{k}^{+}$

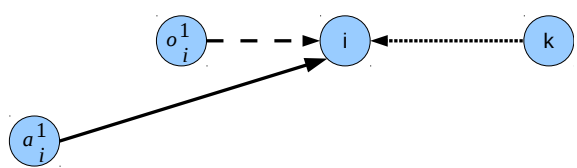

(b) $i$ was not contributing to $\Delta_{k}^{+}$

Fig. 1. The two scenarios for a node $i$ that contributes to $\Delta_{k}^{+}$in Algorithm 2. The old association is drawn with a dashed line, the new one is drawn with a continuous line and the potential association is drawn with a dotted line. 


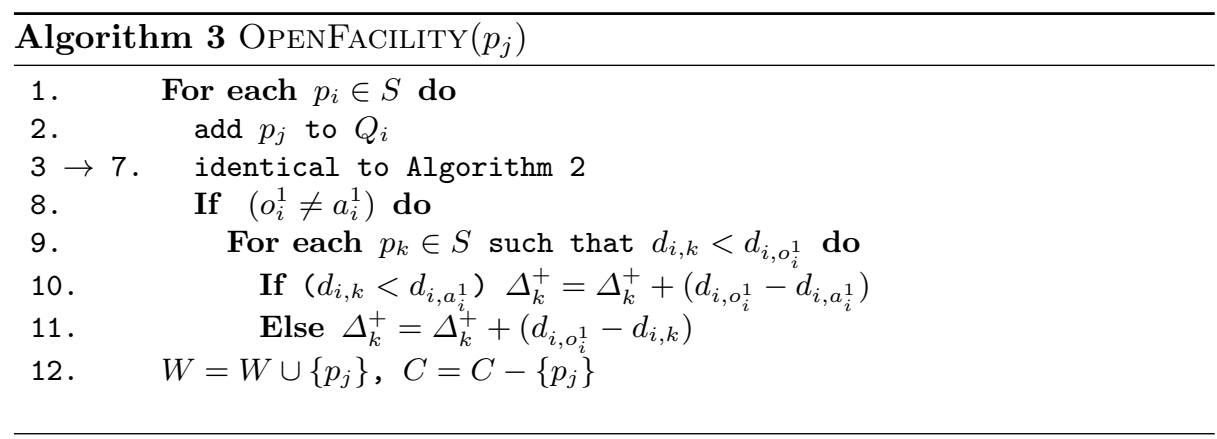

We now consider the complexity of Algorithm 2 for closing a facility. Updating one priority queue is done in $\mathcal{O}(\log (p))$, using an implementation based on heaps, and this has to be done for all points thus Lines 1-2 imply a $\mathcal{O}(m \log (p))$ complexity. Updating $\Delta^{-}$is then done in constant time whereas updating $\Delta^{+}$ is achieved in time linear in the number of points $p_{i}$ whose closest facility has changed. This complexity is optimal as it is necessary to consider all the updated points, and they cannot cancel out since $d_{i, a_{i}^{1}}$ is always increasing $\left(d_{i, a_{i}^{1}} \geq d_{i, o_{i}^{1}}\right)$. The pre-computed lists of points sorted by distance from any other points (Line 9) requires $O\left(\mathrm{~m}^{2}\right)$ space which can be an issue when solving very large problems. However, in practice the cost is dominated by the update of the priority queues [11]. The update of $\Delta^{+}$is costly but only done on a small subset of $S$ whereas the priority queues have to be updated for the $m-p$ points.

\subsection{Opening a Facility}

The update of $\Delta^{-}$is identical to Algorithm 2. The update of $\Delta^{+}$is very similar. As mentioned above, the contribution of $p_{i}$ to $\Delta^{+}$only needs to be updated when $d_{i, a_{i}^{1}}$ is updated, i.e., when $\left(o_{i}^{1} \neq a_{i}^{1}\right)$. However, in this case the contribution of $p_{i}$ to a given $\Delta_{k}^{+}$is reduced either partially or completely since a node is being opened. Line 10 refers to the case where $p_{i}$ remains as a contributor. In this case we just update its contribution by taking into account that $a_{i}^{1}$ is $d_{i, o_{i}^{1}}-d_{i, a_{i}^{1}}$ closer than $d_{i, o_{i}^{1}}$. In Line 11 we remove the contribution of $p_{i}$ completely. Finally, in Line $12, W$ and $C$ are updated accordingly.

\section{A New Incremental Algorithm}

The incremental algorithm presented in the previous section is dominated by the $O(m \log (p))$ cost of updating the priority queues. In practice very few points of $S$ are likely to have a new closest or second closest facility. The left part of Figure 2 shows an example of opening a new facility $p_{j}$. Facilities are indicated by plain circles and points by crosses. The points for which $p_{j}$ is the new closest facility are shown in squares whereas the points for which $p_{j}$ is the new second closest facility are shown in circles. Only a very small number of points of the 

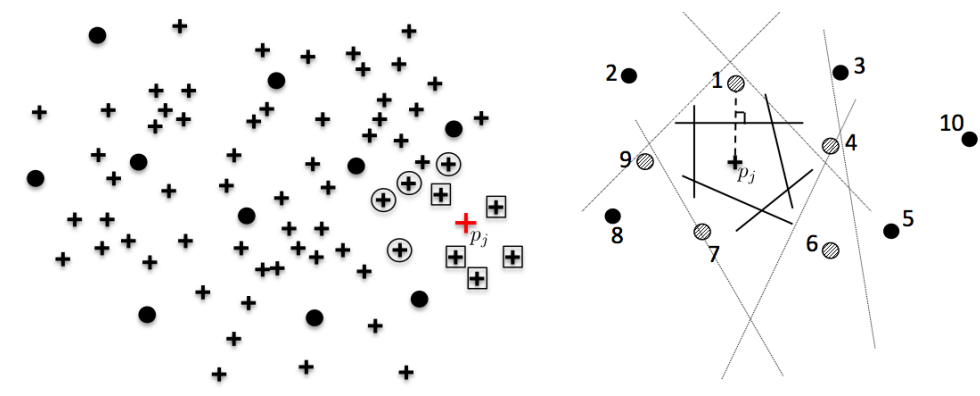

Fig. 2. Example of opening a facility $p_{j}$ on left. Facilities are shown as plain circles, points as crosses and the points having $p_{j}$ as their closest (resp. second closest) facility are shown in a square (resp. a circle). Example of the Voronoi cell of $p_{j}\left(\mathcal{V}\left(p_{j}\right)\right)$ on right. The boundary of the cell is indicated by the dashed nodes so $\mathcal{B}\left(p_{j}\right)=\{1,4,6,7,9\}$.

$m$ points of $S$ are affected. In this paper we focus on approaches that do not maintain the priority queues $Q_{i}$. The set of points for which $a_{i}^{1}$ and $a_{i}^{2}$ need to be maintained, is computed directly using computational geometry techniques.

We begin with a simple approach. We define the radius $r_{j}$ of a facility $j$ as the maximum distance between the facility and any of its points that it covers. The radius of each facility can be maintained easily. If a new facility $j$ is opened then the closest and the second closest of only those points $i$ that are within the reach of $\max _{j \in W}\left(r_{j}\right)$ may change. Using the sorted list of nodes $i$ by increasing distance from node $j$, we only have to iterate over those points $i$ for which $d_{i, j} \leq \max _{j \in W}\left(r_{j}\right)$ rather the complete set $S$.

This approach already takes advantage of Euclidean distance and we will see below how the space and time complexities of Algorithms 2 and 3 can be improved by exploiting computational geometry techniques. Closest point problems are common in computational geometry [14,3]. A strongly related work is [13], which relies on triangulation to speed up the PAM algorithm but does not present complexity results and ignores the optimal incremental schemes that have been developed to improve PAM $[11,16,15,1]$. A more relevant reference is [7] which proposes to improve the k-means algorithm by using geometric reasoning based on kd-trees to speed-up the allocation of each point to its closest cluster. Our work is specific to the p-median/warehouse location problem rather than k-means and the proposed method tries to build upon known optimal incremental algorithms by improving them in the context of Euclidean distances.

\subsection{The Closest Points to a New Facility}

Firstly we focus on updating $a_{i}^{1}$ when opening a new facility. The question we would like to answer efficiently is: determine the points in $S$ which are closer to a given point $p_{j}$ (the facility we would like to open) than to any other points of a given specific set (the facilities $W$ ). This set is precisely characterized by 
the Voronoi cell [3] of $p_{j}$ regarding $W$ denoted $\mathcal{V}\left(p_{j}\right)$. A point $q$ lies in $\mathcal{V}\left(p_{j}\right)$ if and only if $\operatorname{dist}\left(q, p_{j}\right)<\operatorname{dist}\left(q, p_{k}\right)$ for all $p_{k} \in W$. The right part of Figure 2 shows how a Voronoi cell is built. For any two points $p_{j}$ and $p_{k}$ we can define the bisector (see [3], Chapter 7) as the perpendicular bisector of the line segment $\overline{p_{j} p_{k}}$. This bisector splits the plane into two half-planes: one containing the point $p_{j}$ and another containing the point $p_{k} . \mathcal{V}\left(p_{j}\right)$ can be seen as the intersection of the half-planes containing $p_{j}$ obtained by bisecting $\overline{p_{j} p_{k}}$ for all $p_{k} \in W$.

Definition 2 (Boundary of $\left.p_{j}\right)$. The boundary of $p_{j}, \mathcal{B}\left(p_{j}\right)$, is the set of facilities $p_{k}$ such that the bisector of $\overline{p_{j} p_{k}}$ coincides with one of the line segments of the Voronoi cell of $p_{j}$.

Computing one Voronoi cell is based on computing the intersection of $p$ halfplanes which can be done in $O(p \log (p))$ [3]. This, however, does not give us the actual points of $S$ contained in the cell. We propose two approaches to compute the actual points of $S$, the first one is very simple but requires $O\left(m^{2}\right)$ space while the second one remains in $O(m)$ space.

Approach based on the radius. The first approach does not require any special data structure. It is based on the upper bound on the distance between the newly opened facility $j$ and the nodes which will have $j$ as their facility. The Voronoi cell of $p_{j}$ is a convex polygon which is associated with a set of (corner) points. The minimum and the maximum distances between $j$ and any of the corner points of the Voronoi cell is denoted by $r_{\min }$ and $r_{\max }$ respectively. Any point whose distance from $j$ is less than $r_{\min }$ will definitely have $j$ as its new facility. Any point whose distance from $j$ is more than $r_{\max }$ will not be affected by the new facility. Any point whose distance from $j$ is between $r_{\max }$ and $r_{\min }$ could possibly be affected. Therefore one has to iterate over all the points $i$ whose distance from $j$ is less than or equal to $r_{\max }$. This is easy if we have access to the points sorted in the increasing distance from $j$ which requires $O\left(\mathrm{~m}^{2}\right)$ space.

Approach based on a kd-tree. The second approach is based on the use of a common data structure in computational geometry, namely, a kd-tree [2]. A kdtree for two dimensional data points is a binary tree data structure where at each node the space is partitioned horizontally or vertically. The two children of each node correspond to two regions of the space.

The kd-tree is built once and contains the points of $S$, the subdivision of the space is made alternatively on the $x$ and $y$ coordinates of a point of $S$ and continues until all regions contain no more than one point. A balanced kd-tree (a tree such that each leaf node is more or less at the same distance to the root) can be easily built by inserting the points in a specific order, simply by choosing at each step the median of the points sorted on the corresponding coordinate. In a balanced kd-tree, obtaining one point of $S$ contained in a rectangular area (a range query parallel to the axis used for partitioning the space in the kd-tree) can be done in $O(\sqrt{m})$ and finding all points of $S$ contained in a rectangular area costs $O(\sqrt{m}+k)$, where $k$ is the number of points in the corresponding area [3]. 
The tree is traversed as long as the region of a node intersects the area. When a leaf is reached, the corresponding point is added in the list of points to return. Similarly, when the area fully contains the region of a node, all points contained in the subtree rooted at this node are added in the answer to the query.

When the area of the Voronoi cell is not a rectangle but an arbitrary convex polygone, checking the intersection with the rectangular region of a node can be done in $O(h)$ time where $h$ is the size of the boundary of the cell, i.e., $h=\left|\mathcal{B}\left(p_{j}\right)\right|$. However, in this latter case, the $O(\sqrt{m})$ is not guaranteed. In order to express the complexity, we consider the enclosing rectangle of the Voronoi cell as the query. Let $k$ be the number of points in the cell. The Voronoi cell can be enclosed in a rectangle containing $k^{\prime}$ points $\left(k^{\prime}>k\right)$ in which case the overall complexity is $O\left(p \log (p)+\sqrt{m}+k^{\prime}\right)$. In practice we apply the algorithm using the Voronoi cell itself to obtain more pruning in the tree.

\subsection{Updating the Two Closest Points when Opening a Facility}

We now focus on updating $a_{i}^{1}$ and $a_{i}^{2}$ when opening a new facility. We extend the previous idea to find the set of points that have either a new closest or a new second closest facility. The question we would like to answer efficiently is: determine the points in $S$ for which a given point, $p_{j}$ (the facility we would like to open), is one of their two closest neighbors regarding a given specific set (the facilities $W$ ). Determining such a set exactly is slightly harder since the points of the set may not necessarily be enclosed in a convex polygon. Characterizing such a set involves the computation of the Voronoi cell of each facility of $\mathcal{B}(j)$, which will increase the complexity.

We generalize the previous ideas so that the same scheme applies by replacing the concept of Voronoi cell with a set $\mathcal{V}^{\prime}\left(p_{j}\right)$ containing the set of points $q$ for which $p_{j}$ is closer than their second closest neighbor in $W$. In order to do so we suggest a simple convex approximation based on the concept of Extended Voronoi cell.

Definition 3 (Extended Voronoi cell). Given a point $p_{j}$, the extended Voronoi cell $\mathcal{V}_{2}\left(p_{j}\right)$ is defined as the Voronoi cell of $p_{j}$ associated with the set of facilities $W-\mathcal{B}\left(p_{j}\right)$.

Figure 3 illustrates an extended Voronoi cell. Similarly the concept of boundary can be extended and we will denote $\mathcal{B}_{2}\left(p_{j}\right)$ the boundary of the extended Voronoi cell of $p_{j}$.

Lemma $1\left(\mathcal{V}^{\prime}\left(p_{j}\right) \subseteq \mathcal{V}_{2}\left(p_{j}\right)\right)$.

Proof. Consider a point $q$ outside of $\mathcal{V}_{2}\left(p_{j}\right) . q$ is closer to a facility $p_{k} \in W-\mathcal{B}\left(p_{j}\right)$ than to $p_{j}$ because $\mathcal{V}_{2}\left(p_{j}\right)$ is the Voronoi cell regarding $W-\mathcal{B}\left(p_{j}\right) . q$ is also necessarily closer to a point of $\mathcal{B}\left(p_{j}\right)$ than to $p_{j}$ since $q$ does not belong to $\mathcal{V}\left(p_{j}\right)$. Thus $p_{j}$ cannot be one of the two closest neighbors of $q$.

Notice that $\mathcal{V}^{\prime}\left(p_{j}\right) \neq \mathcal{V}_{2}\left(p_{j}\right)$. For example in Figure 3 (left), the area paved with squares within $\mathcal{V}_{2}\left(p_{j}\right)$ contains points that are closer to 4 and 6 than to $p_{j}$. 

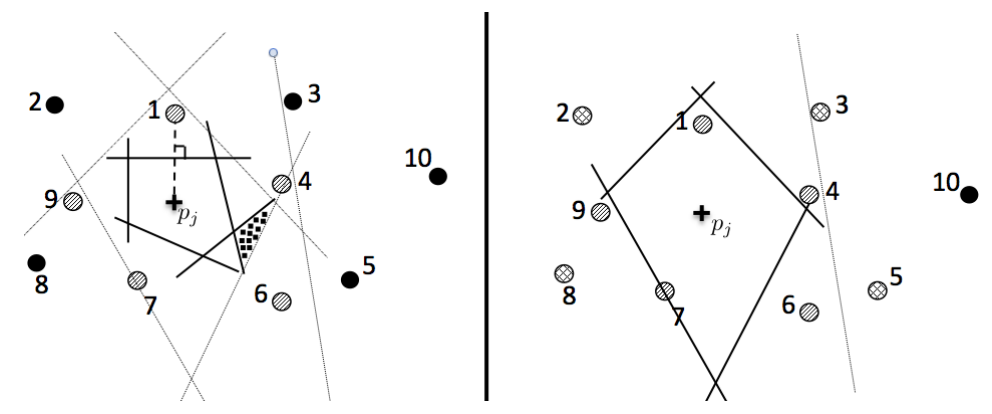

Fig. 3. On the left : Example of $\mathcal{V}\left(p_{j}\right)$ the Voronoi cell of $p_{j}$, the boundary of cell is defined by the dashed nodes so $\mathcal{B}\left(p_{j}\right)=\{1,4,6,7,9\}$. On the right: Example of $\mathcal{V}_{2}\left(p_{j}\right)$ the extended Voronoi cell of $p_{j}$ whose boundary $\mathcal{B}_{2}\left(p_{j}\right)=\{2,3,5,8\}$.

\subsection{Updating the Two Closest Points when Closing a Facility}

We consider how to update $a_{i}^{1}$ and $a_{i}^{2}$ when closing a facility $p_{j}$. Similar to the previous case, the set of points that have $p_{j}$ as their closest or second closest facility can be computed using the extended Voronoi cell of $p_{j}$. In this case however we can assume that we maintain the set of points connected to $p_{j}$ in a dedicated data structure, e.g. a list. When closing $p_{j}$, the closest or second closest facility of these points has to be updated. A simple solution would be to iterate over the current opened facilities $W$ to find the two closest. Alternatively, this is exactly a 2-nearest neighbors problem. One seeks to quickly identify the two nearest neighbors in $W$ of the points that were connected to $p_{j}$. The k-nearest neighbors is a classic problem in machine learning [2] and efficient implementations rely on the use of kd-trees $[2,4]$. Assuming that we maintain a kd-tree for the set $W$, finding the two nearest neighbors of a given point can be done efficiently in a balanced kd-tree. The worst-case complexity remains $O(p)$ as it is easy to construct examples where all the leaves of the tree will have to be checked. The complexity analysis presented in [4] reports that the expected number of nodes inspected in the tree is in $O(\log (p))$.

\subsection{Updating Algorithms 2 and 3}

The complexity reported for the following algorithms does not include the complexity due to maintaining $\Delta^{-}$and $\Delta^{+}$which is optimal [11] and linear in the

number of changes of closest or second closest. We introduce three additional data structures:

1. $S_{j}$, corresponding to the list of nodes for which facility $p_{j}$ is either the closest or second closest facility.

2. $K W$ is a kd-tree of the set $W$ of facilities. $K W$ is therefore dynamic and must be updated when closing/opening facilities.

3. $K S$ is a kd-tree of the set $S$ of nodes. $K S$ is static and pre-computed initially. 


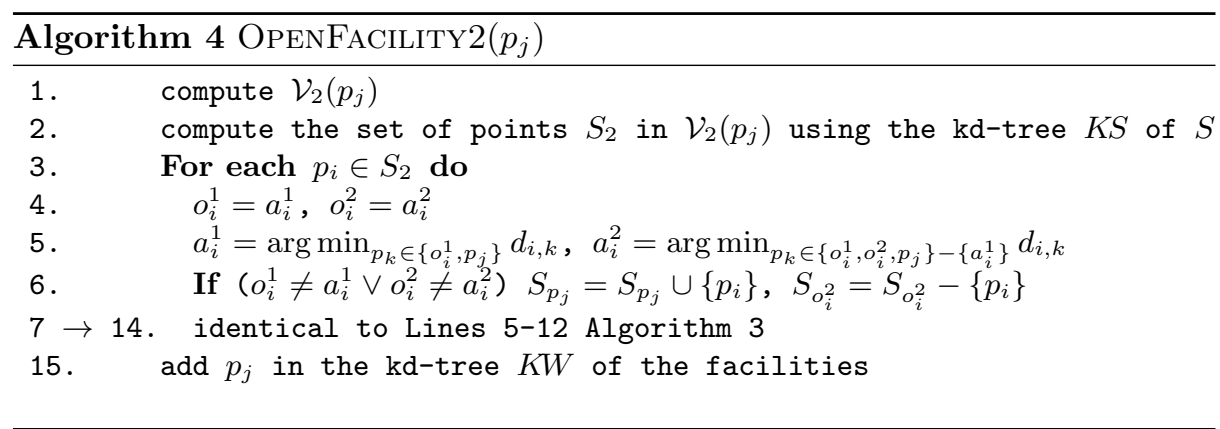

Algorithm 4 is the new version of Algorithm 3 taking advantage of the ideas based on computational geometry. The extended Voronoi cell of the facility opened is computed first (Line 1) and the points contained in the cell $\left(S_{2}\right)$ are extracted using the kd-tree $K S$ of $S$ (Line 2). The loop over all the points of $S$ is replaced by a loop over the points contained in $S_{2}$. The closest or second-closest facility of $p_{i}$ might now be $p_{j}$ but this update takes constant time; so does the update of the $S_{j}$ data structure, which is useful for Algorithm 5 when closing a facility. Finally, the incremental maintenance of $\Delta^{-}$and $\Delta^{+}$remain unchanged.

Line 1 takes $O(p \log (p))$, Line 2 (assuming we are using the enclosing rectangle of the Voronoi cell) takes $O\left(\sqrt{m}+k^{\prime}\right) . k^{\prime}$ is the number of points in the enclosing rectangle so it is greater than the number of points contained in the cell $\left(k^{\prime} \geq k\right)$. Finally Line 5 is performed in $O(1)$ and the update of $K W$ (line 15) is done in $O(\log (p))$. The complexity of Algorithm 4 is $O\left(p \log (p)+\sqrt{m}+k^{\prime}\right)$. We recall that the complexity of the previous incremental algorithm is dominated by the $O(m \log (p))$ factor which involves examining systematically all the $m$ points. Algorithm 4 does not have this drawback as $m$ does not appear directly in the complexity but only in a worst case where $k^{\prime}=m$. In practice, we expect $k^{\prime}$ to be much smaller than $m$.

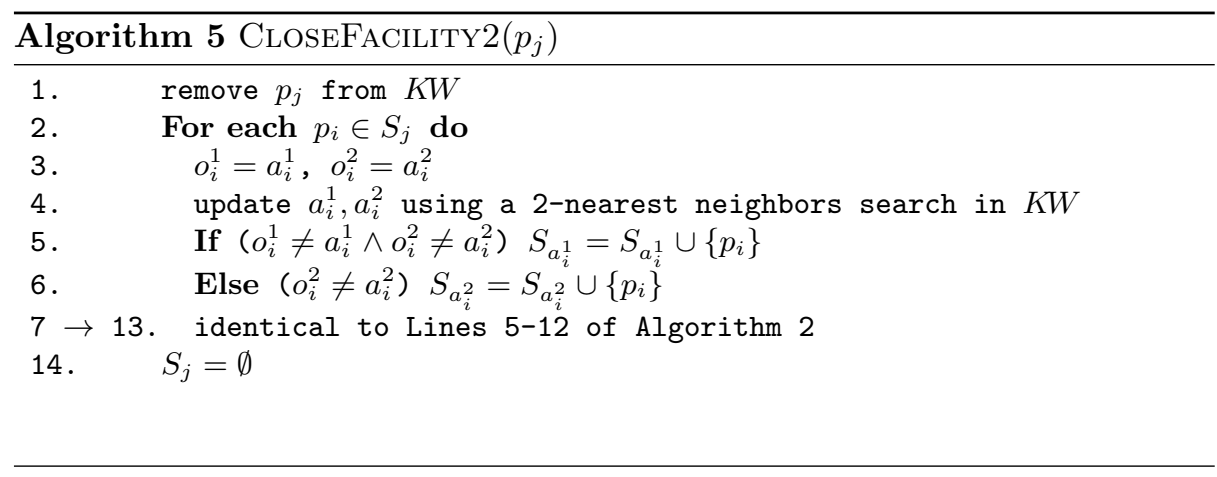


Similarly, Algorithm 5 is the new version of Algorithm 2. The list $S_{j}$ is used to iterate over the points which had $p_{j}$ as their closest or second closest. The only difference is that the update of the $a_{i}^{1}$ and $a_{i}^{2}$ is done by using the kd-tree of the facilities $K W$ since we no longer maintain the priority queues. The worstcase complexity of the nearest neighbors search in a balanced kd-tree is $O(p)$ but its expected complexity is $O(\log (p))$. Note that $K W$ has to be re-balanced from time to time to guarantee this complexity. The update of the kd-tree $K W$ is done in Line 1 and takes $O(\log (p))$ so that the overall expected complexity for closing a facility is $O(k \log (p))$ with $\left|S_{j}\right|=k$.

\section{Time and Space Complexities}

We can distinguish three different approaches:

- BL (Base Line): the approach proposed in [11] corresponding to Algorithms 2 and 3 that iterates over all points, updates the priority queues as well as the two closest facilities of each point when needed.

- LIBL (Less Incremental Base Line): this approach is a simple modification of the BL that does not use geometry. It simply ignores the priority queues in Algorithm 3 as the two closest facilities can be updated in constant time when opening a facility and it is based on Algorithm 5 for closing a facility but does not use the kd-tree $K W$ to update the two closest facilities. It simply iterates over $W$ to update the two closest facilities.

- GEO (Geometric): the new approach proposed based on Algorithm 4 and 5.

We summarize the complexity of the three approaches in Tables 1 and 2 where $m$ is the number of nodes, $p$ the number of facilities, $k$ the number of nodes which have $p_{j}$ as a closest or second closest, and $k^{\prime}$ is an upper bound on $k$ useful to express the complexity (it is the number of points contained in the enclosing rectangle of the extended Voronoi cell) as we still have $k^{\prime} \leq m$; ignoring the linear update of $\Delta^{-}$and $\Delta^{+}$which is identical in the three cases.

The space complexity is dominated by the pre-computed lists of points sorted by distance (refered as proximity lists) from any other points (Line 9 of Algorithms 3 and 2) which requires $O\left(\mathrm{~m}^{2}\right)$ space. This is an issue for solving large problems. It can be overcomed in the case of GEO as it is simply a query in the kd-tree $K S$ where we seek all the points contained in a circle, centered in $p_{i}$, of radius $d_{i, a_{i}^{1}}$ (Algorithm 2) or $d_{i, o_{i}^{1}}$ (Algorithm 3). Various mechanisms can be explored for BL and LIBL such as storing only a fixed limited number of points in the lists and relying in an $O(m)$ search in the worst case but we loose the optimal update of $\Delta^{-}$and $\Delta^{+}$. So overall the proximity lists require $O\left(m^{2}\right)$ space,

Table 1. Summary of time complexities of the different schemes

\begin{tabular}{|c|r|r|r|}
\hline operation & BL & LIBL & GEO \\
\hline open a facility $p_{j}$ & $O(m \log (p))$ & $O(m)$ & $O\left(p \log (p)+\sqrt{m}+k^{\prime}\right)$ \\
\hline close a facility $p_{j}$ & $O(m \log (p))$ & $O(k p)$ & expected $: O(k \log (p))$, worst-case: $O(k p)$ \\
\hline
\end{tabular}


Table 2. Summary of space complexities of the different schemes

\begin{tabular}{|r|r|r|r|}
\hline & BL & LIBL & GEO \\
\hline space complexity & $O\left(m^{2}+m p\right)$ & $O\left(m^{2}\right)$ & $O(m+p)$ \\
\hline
\end{tabular}

the priority queues are consuming $O(m p)$, the various data structures $a, \Delta$ and $S$ are all in $O(m)$ and the two kd-trees, $K S$ and $K W$, need respectively $O(m)$ and $O(p)$ space. Notice that GEO is the only scheme that is scalable in memory with $m$ and $p$, in practice.

\section{Empirical Study}

The experiments are organized into two sets. ${ }^{2}$ In the first set of experiments we investigate the behavior of the proposed algorithms on randomly generated data. In particular we focus in these experiments on benchmarking the two algorithms openFacility and closeFacility. The algorithms compared are identical regarding the incremental update of $\Delta^{+}$and $\Delta^{-}$. They only differ in the way that the closest and second closest facilities are identified and maintained. Therefore, the time reported when comparing GEO, BL and LIBL are only considering the update of the closest and second closest facilities. Instances are randomly generated by uniformly drawing points in the plane with $x$ and $y$ in $[0,10000]$. For each $m$ in $\{2000,5000,10000,20000,40000,80000\}$ ten instances were generated $^{3}$. We performed 100 runs for each instance and each value of $p$. For each $m$ we consider four values of $p$, which are defined as percentages of $m$ : $0.1 \%, 1 \%, 2.5 \%$ and $5 \%$. Each run consists of picking $p$ random points out of the $m$ points to define the set $W$, and a single random extra point: $p_{j} \notin W$ when the facility is being opened or $p_{j} \in W$ when it is being closed. In each run we measure the time needed by the different approaches: BL, LIBL and GEO. For each pair $(m, p), 1000$ queries are performed overall (100 on each of the 10 instances) and the cumulative times are reported.

As shown in Figures 4(a) and 4(b), the time of GEO increases moderately with $m$ in practice. This is particularly true in the closeFacility case (see Figure 4(b)). The increase observed in the openFacility case is mainly due to the increase of points in the cell that need to be processed as $m$ increases. In Figures 4(c) and 4(d), we also observed that LIBL does not seem to be significantly affected by the size of $p$, which suggests that the advantage of GEO over LIBL reduces when $p / m$ increases. BL cannot scale any further because of memory issues. Notice that the priority lists are consuming $O(m p)$ space, which becomes too big when $m=80000$.

In the second set of experiments we considered eight SCP cases. Table 3 shows the parameters of each case. Half of the cases correspond to real cases

\footnotetext{
${ }^{2}$ Experiments were run on Linux 2.6.25 x64 on a Dual Quad Core Xeon CPU machine with overall $11.76 \mathrm{~GB}$ of RAM and processor speed of $2.66 \mathrm{GH}$.

${ }^{3}$ The large sizes considered would be more relevant for p-median problems than for warehouse location problems.
} 


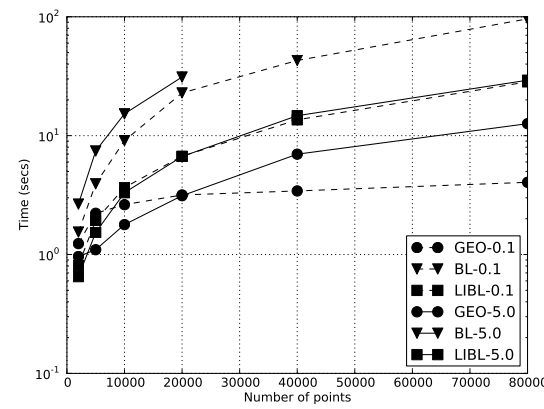

(a) Open - p fixed (0.1 and 5).

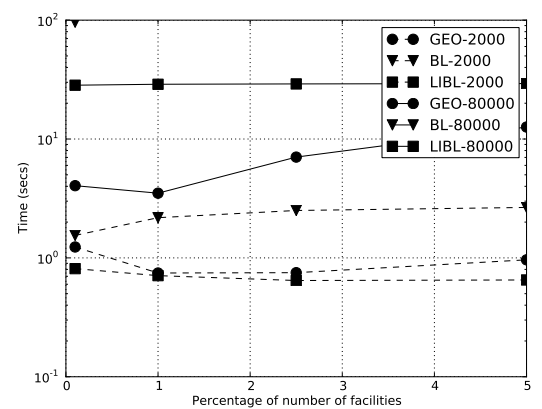

(c) Open - m fixed (2000 and 80000).

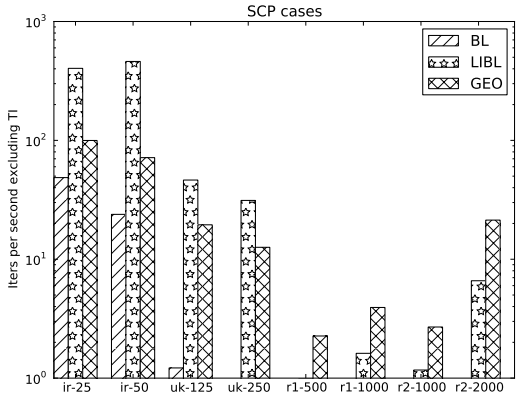

(e) Number of iterations per second.

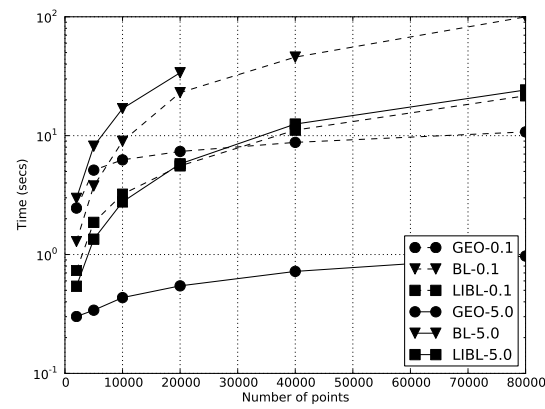

(b) Close - p fixed (0.1 and 5).

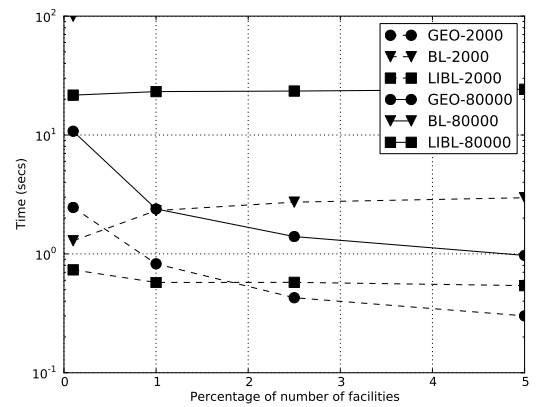

(d) Close - m fixed (2000 and 80000).

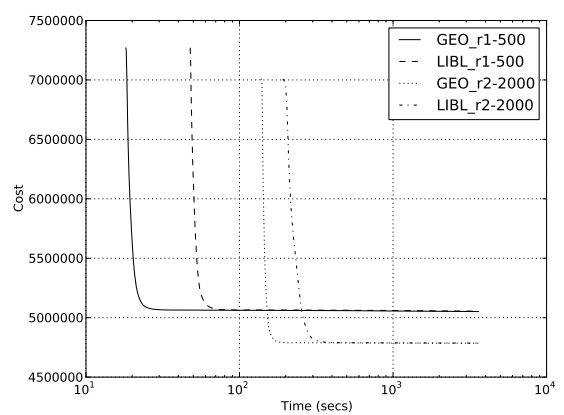

(f) Evolution of the quality of the solution.

Fig. 4. A summary of the results from our empirical evaluation

coming from network operators in Ireland and UK. The second half corresponds to cases that were randomly generated. In the real case there is only one instance. In the random cases 10 instances were generated. For each instance we had 10 runs. The values reported in each case correspond to the average on all the instances over all runs. For each run we considered a threshold of 3600 
Table 3. SCP cases

\begin{tabular}{|l|r|r|r|r|r|r|r|r|}
\hline & ir-25 & ir-50 & uk-125 & uk-250 & r1-500 & r1-1000 & r2-1000 & r2-2000 \\
\hline$m$ & 1100 & 1100 & 5390 & 5390 & 20000 & 20000 & 40000 & 40000 \\
\hline$p$ & 25 & 50 & 125 & 250 & 500 & 1000 & 1000 & 2000 \\
\hline source & real & real & real & real & random & random & random & random \\
\hline
\end{tabular}

seconds. We observed the approaches spend a significant amount of time in the initialization of the data structures which takes takes place each time we restart our current implementation. We restart if after a specified number of iterations no improvement of the best known solution has been seen. In order to have a fair measure of the speed (i.e., number of iteration per second) of each approach, we discounted the initialization time when computing the speed. Figure 4(e) shows the comparison of the approaches with respect to the speed. We can see that, even though LIBL performs quite well in the medium-size cases, GEO is the faster approach when $m$ is big. When looking at the evolution of the quality of the solution we observe that the approaches tend to converge quite rapidly. In Figure 4(f) we only show random cases since the situation of the real cases is even more extreme. The difference observed between the approaches is mostly due to the time they spend in finding the first solution, which is basically the time spent in the first initialization.

\section{Conclusion}

We have presented a novel approach to achieve efficient and incremental evaluation of the neighborhood of local search algorithms for facility location problems. These ideas apply when the cost for allocating a client to a facility is proportional to the Euclidean distance. We showed how to use computational geometry to efficiently maintain the closest or second closest client to a facility. We also showed how this can be integrated within existing state of the art local search techniques for this class of problems. Any neighborhood involving the maintenance of the two closest points could benefit from these ideas and the techniques presented make sense for a constraint-based local search framework where this type of incrementality is needed for spatial location problems. We presented empirical results that demosntrated the utility of our approach ${ }^{4}$.

Many improvements are possible as computational geometry is a very rich and active domain. For example, the use of range trees [3] instead of kd-trees would lead to a $O\left(\log ^{2}(m)+k\right)$ complexity (instead of $\left.O(\sqrt{(} m)+k\right)$ for kd-tree) for a small increase of the space complexity to $O(m \log (m)$ ) (instead of $O(m)$ for the kd-tree). We will explore the generalization of this work to other metric distances or general distance matrices.

\footnotetext{
${ }^{4}$ Our focus has been on local search approaches to SCP. However, we are certainly aware of heuristic approaches to tackle SCP [12]. We are currently considering these types of approaches too.
} 


\section{References}

1. K. S. Al-Sultan and M. A. Al-Fawzan. A tabu search approach to the uncapacitated facility location problem. Annals of Operations Research, 86:91-103, 1999.

2. Jon Louis Bentley. Multidimensional binary search trees used for associative searching. Commun. ACM, 18:509-517, September 1975.

3. M. de Berg, O. Cheong, M. van Kreveld, and M. Overmars. Computational Geometry, Algorithms and Applications. Springer-Verlag, 2008.

4. Jerome H. Friedman, Jon Louis Bentley, and Raphael Ari Finkel. An algorithm for finding best matches in logarithmic expected time. ACM Trans. Math. Softw., 3:209-226, September 1977.

5. L.L. Gao and E.P. Robinson. Uncapacitated facility location: General solution procedures and computational experience. European Journal of Operations Research, 76:410-427, 1994.

6. J. Han, M. Kamber, and A. K. H. Tung. Spatial Clustering Methods in Data Mining: A Survey. Taylor and Francis, 2001.

7. Tapas Kanungo, David M. Mount, Nathan S. Netanyahu, Christine D. Piatko, Ruth Silverman, and Angela Y. Wu. An efficient k-means clustering algorithm: Analysis and implementation. IEEE Trans. Pattern Anal. Mach. Intell., 24:881-892, July 2002.

8. Leonard Kaufman and Peter J. Rousseeuw. Finding Groups in Data: An Introduction to Cluster Analysis (Wiley Series in Probability and Statistics). WileyInterscience, March 2005.

9. Jozef Kratica, Dusan Tosic, Vladimir Filipovic, Ivana Ljubic, and Communicated P. Tolla. Solving the simple plant location problem by genetic algorithm. RAIRO Operations Research, 35:127-142, 2001.

10. Deepak Mehta, Barry O'Sullivan, Luis Quesada, Marco Ruffini, David Payne, and Linda Doyle. Designing resilient long-reach passive optical networks. In IAAI, 2011.

11. Laurent Michel and Pascal Van Hentenryck. A simple tabu search for warehouse location. European Journal of Operational Research, 157(3):576-591, September 2004 .

12. Kyriakos Mouratidis, Dimitris Papadias, and Spiros Papadimitriou. Tree-based partition querying: a methodology for computing medoids in large spatial datasets. VLDB J., 17(4):923-945, 2008.

13. Zhang Qiaoping and Couloigner Isabelle. A new and efficient k-medoid algorithm for spatial clustering. Computational Science and Its Applications, ICCSA 2005, 3482:207-224, 2005.

14. Michael Ian Shamos and Dan Hoey. Closest-point problems. In Proceedings of the 16th Annual Symposium on Foundations of Computer Science, pages 151-162, Washington, DC, USA, 1975. IEEE Computer Society.

15. Minghe Sun. Solving the uncapacitated facility location problem using tabu search. Computers and Operations Research, 33(9):2563-2589, 2006.

16. R.A Whitaker. A fast algorithm for the greedy interchange of large-scale clustering and median location problems. INFOR, (21):95-108, 1983. 